\title{
GRAPHICAL REPRESENTATIONS OF MEMBERSHIP FUNCTIONS OF MAXIMUM AND MINIMUM OF TWO FUZZY NUMBERS USING COMPUTER PROGRAM
}

\author{
Shapla Shirin ${ }^{1}$ \\ Department of Mathematics, University of Dhaka \\ E-mail: shapla@univdhaka.edu \\ and \\ Goutam Saha \\ Department of Mathematics, University of Dhaka \\ E-mail: ranamath08@univdhaka.edu
}

Received 10.12.2010

Accepted 26.02.2012

\begin{abstract}
The set of real numbers $\mathbb{R}$ is linearly ordered, but in the fuzzy set theory, this relation is true only for some set of fuzzy numbers where the sets of fuzzy numbers are expressed as the linguistic variables. Different types of Fuzzy machines based on fuzzy logic have been invented where fuzzy logics are described by fuzzy numbers and the fuzzy numbers are needed to compare. Besides these, many techniques are available to assist decision-makers to compare different fuzzy numbers. For these reasons, it is necessary to compute the maximum and the minimum of fuzzy numbers. Till now many researchers introduced different methods for computation, which are done by hand calculation, but these are very disgusting and time consuming to us. In this paper, we presents an algorithm to compute the maximum and the minimum of any two triangular fuzzy numbers, so that one can compare two fuzzy numbers easily in a short time and visualize the analytic expressions and the graphical representations of the maximum and the minimum of any two triangular fuzzy numbers. By using CAS (MATHEMATICA 7.0), the algorithm is implemented in a computer program in order to do these. This algorithm can easily be extended to apply for any type of fuzzy numbers which are comparable. Even it is able to compare more than two fuzzy numbers by comparing the maximum fuzzy number or minimum fuzzy number with another new fuzzy number.
\end{abstract}

Keywords : Fuzzy numbers, Maximum fuzzy numbers (MAX), and minimum fuzzy numbers (MIN), Graphical representation using computer program, Comparable fuzzy numbers.

\section{Introduction}

Researchers are supposed to use fuzzy numbers in some applications to solve the real life problem. Over the Years Fuzzy Set Theory has been playing an important role to deal with the problems having uncertainty, vagueness, doubtful data and so on, which cannot be solved with the help of available classical methods. In1965, Zadeh [9] introduced the

${ }^{1}$ Author of Correspondence : shapla@ univdhaka.edu. 
concept of fuzzy set theory. In 1978, Dubois and Prade defined the fuzzy numbers as a fuzzy subset of the real line $[1-6,8]$. Fuzzy numbers are used in statistics, computer programming, engineering (especially communications), experimental science and also in mathematics. In all of these fuzzy types of presentation, fuzzy numbers are most used as in linguistic, decision making, knowledge representation, medical diagnosis, control systems, databases, and so forth. A special case of a fuzzy number is an interval. Viewed in this perspective, operations on fuzzy numbers may be viewed as a generalization of operation on interval. We consider a fuzzy number to be an extension of the concept of the interval of confidence.

We know that the set of real numbers is linearly ordered, that is two real numbers are comparable with the relation 'less or equal to', and this is done by the operators 'max' or 'min'. These operators cannot be applied to the set of fuzzy numbers. In this case we use the operators 'MAX' and 'MIN'. In fuzzy set theory, the set of fuzzy numbers are not linearly ordered though maximum (MAX) and minimum (MIN) of two fuzzy numbers can be computed. However, some of the sets of fuzzy numbers are linearly ordered, for example, in the set of fuzzy numbers 'performance $=\{$ very small, small, medium, large, very large\}, two fuzzy numbers are comparable[6]. This set is named as fuzzy linguistic variable and these types of variables are used to a decision making problem or machine. It is also essential to compare dates of excavation data, represented by fuzzy numbers, to obtain archaeological simulated maps[7], etc.

In order to that it is important to compute the maximum and the minimum of two fuzzy numbers. HONG and KIM [4] proposed a method to compute $\alpha$ - cuts of the maximum and the minimum of more than two continuous and non-continuous fuzzy numbers via $\alpha-$ cut presentation. But it is time consuming to get the result using [4]. After that an easy method has been introduced in [7], which are able to compute the membership functions of the maximum and the minimum of more than two triangular fuzzy numbers by hand calculation. This method is comparatively better than before, but it also takes time.

In this paper, our goal is to introduce an algorithm and create a computer program to compute and visualize the maximum operator (MAX) and the minimum operator (MIN) of two triangular fuzzy numbers, which can produce the analytic expressions and the corresponding graphical representations of the maximum and the minimum of two triangular fuzzy numbers in a shortest possible time. This algorithm can easily be extended for two trapezoidal shaped or any other fuzzy numbers. Here it is noted that this algorithm is able to compare more than two fuzzy numbers by considering the maximum of two fuzzy numbers $M A X\left(\mu_{r} \gamma\right)$ or the minimum of two fuzzy numbers $M I N\left(\mu_{r} \gamma\right)$ as a fuzzy number with another new fuzzy number repeatedly.

\section{Preliminaries}

In this section some definitions have been given for better understanding of the contains of the next sections. A fuzzy number is defined by the membership function $\mu$ which is defined on the set of real numbers $\mathbb{R}$. 
Definition 2.1: A fuzzy set $\mu: \mathbb{R} \rightarrow \mathrm{I}[0,1)$ is said to be a fuzzy number if it satisfies the following properties :

(a) $\mu$ is a normal fuzzy set;

(b) for every $\alpha \in(0,1]$, the $\alpha-c u t$ of $\mu$, ${ }^{a} \mu$, is a closed interval denoted by $[\underline{\mu}(\alpha), \bar{\mu}(\alpha)]$

(c) the support of $\mu,{ }^{0 *} \mu$, is bounded.

Definition 2.2: The triangular fuzzy number $\mu: \mathbb{R} \rightarrow \mathrm{I}[0,1)$ is defined as under:

$$
\mu(x)=\left\{\begin{array}{ccc}
0 & \text { if } & x \leq \alpha_{a} \text { and } x \geq \alpha_{b} \\
\frac{x-a_{a}}{a_{c}-a_{a}} & \text { if } & \alpha_{a}<x<a_{c} \\
1 & \text { if } & x=a_{c} \\
\frac{a_{b}-x}{a_{b}-a_{c}} & \text { if } & \alpha_{c}<x<\alpha_{b}
\end{array}\right.
$$

We shall denote the set of all fuzzy numbers by $\mathbb{R}$.

Definition 2.3 : Let $A=\left[a_{n} b\right]$, and $B=\left[c_{s} d\right]$ be two closed interval. Then, the maximum and minimum of the closed intervals, which are also closed interval and denoted by $M A X\{A, B\}$ and $M I N(A, B)$, respectively, are defined by

(a) $\operatorname{MAX}\{A, B\}=[\max \{a, c\},, \max \{b, d\}]$;

(b) $M I N\{A, B\}=[\min \{a, c\},, \min \{b, d\}]$,

where 'max', and 'min' are used for the maximum and minimum of real numbers, respectively.

The operators $M A X$ and $M I N$, which are computed via $\alpha-$ cut presentation, are the operations which are used to compare two fuzzy numbers [5].

Definition 2.4 : Let $\mu_{1}, \mu_{2} \in \mathbb{R}$ and $\alpha \in I=[0,1]$. Then, For all $x_{1}, x_{2}, z \in \mathbb{R}$, Suppose that $\alpha_{\mu}=[\mu(\alpha), \bar{\mu}(\alpha)]$ and ${ }^{*} \gamma=[\gamma(\alpha), \bar{\gamma}(\alpha)]$ are the $\alpha$-cuts of $\mu$ and $\gamma$, respectively. Then, the maximum and the minimum of $\varepsilon_{\mu}$ and ${ }^{*} \gamma$, denoted by ${ }^{\alpha}\left[M A X\left(\mu_{s} \gamma\right)\right]$ and ${ }^{\alpha}\left[M I N\left(\mu_{s} \gamma\right)\right]$ respectively, are defined by

(a) ${ }^{a}[\operatorname{MAX}(\mu, \gamma)]=\left[\operatorname{MAX}\left\{{ }^{\alpha_{\mu},}{ }^{a} \gamma\right\}\right]$;

(b) ${ }^{\alpha}[\operatorname{MIN}(\mu, \gamma)]=\left[\operatorname{MIN}\left({ }^{{ }}{ }_{\mu,}{ }^{\alpha} \gamma\right)\right]$.

Definition 2.5 : Two fuzzy numbers $\mu$ and $\gamma$ are said to be comparable if and only if $\mu$ is less or equal to $\gamma$ (in sense of fuzzy subset). 


\section{Algorithm to Compute and Visualize $M A X$ and $M I N$ of Two Fuzzy Numbers}

An algorithm is presented to obtain and visualize the membership functions $\operatorname{MAX}(\mu, \gamma)$ and $M I N\left(\mu_{i} \gamma\right)$ of the maximum and the minimum of two triangular fuzzy numbers. It is then implemented in a computer program which is able to visualize the analytic expressions and the graphical representation of the membership functions $\operatorname{MAX}(\mu, \gamma)$ and $M I N\left(\mu_{r} \gamma\right)$. Now, it is easy to compare between two triangular fuzzy numbers.

The algorithm is given below :

Step.1. Input the fuzzy numbers $\mu$ and $\gamma$.

Step.2. Compute $\alpha_{\mu}=[\underline{\mu}(\alpha), \bar{\mu}(\alpha)]$ and $\alpha_{\gamma}=[\underline{\gamma}(\alpha), \bar{\gamma}(\alpha)]$.

Step.3. Compute all possible intersecting points for $\alpha=\alpha_{1}, \alpha_{2,}, \alpha_{3}$ of $\mu(x)$ and $\gamma(x)$.

Step.4. Obtain the open intervals $\left(0, \alpha_{1}\right),\left(\alpha_{1}, \alpha_{2}\right),\left(\alpha_{2}, \alpha_{3}\right),\left(\alpha_{3}, 1\right)$.

Step.5. Using Definition 2.4, compute ${ }^{\alpha}\left[M A X\left(\mu_{,} \gamma\right)\right]$ and ${ }^{a}\left[M I N\left(\mu_{s} \gamma\right)\right]$ in the intervals $\left(0, \alpha_{1}\right),\left(\alpha_{1}, \alpha_{2}\right),\left(\alpha_{2}, \alpha_{3}\right),\left(\alpha_{3}, 1\right)$.

Step.6. Compute the membership functions $\operatorname{MAX}(\mu, \gamma)(x)$ and $M I N(\mu, \gamma)(x)$; and the corresponding $x$ - values for the intervals $\left(0, \alpha_{1}\right),\left(\alpha_{1}, \alpha_{2}\right),\left(\alpha_{2}, \alpha_{3}\right),\left(\alpha_{3}, 1\right)$.

Step.7. Sketch the membership functions $M A X(\mu, \gamma)(x)$ and $M I N(\mu, \gamma)(x)$.

By using this algorithm, $M A X\left(\mu_{i} \gamma\right)(x)$ and $M I N\left(\mu_{i} \gamma\right)(x)$ are determined with the help of hand calculations which are shown in the following examples. There are four cases which are depending on maximum number of intersecting points of $\mu(x)$ and $\gamma(x)$, since there are only three possible intersecting points. So, the four examples are considered for no intersecting points, for one intersecting point, for two intersecting points, and for three intersecting points, respectively.

Example 3.1 : We compute the maximum and the minimum of two fuzzy numbers $\mu$ and $\gamma$ which are defined by

$\mu(x)=\left\{\begin{array}{ccc}x-1 & \text { if } & 1 \leq x \leq 2 \\ 3-x & \text { if } & 2 \leq x \leq 3 \\ 0 & \text { if } & x<1 \text { and } x>3\end{array} ; \quad \gamma(x)=\left\{\begin{array}{ccc}x-4 & \text { if } & 4 \leq x \leq 5 \\ 6-x & \text { if } & 5 \leq x \leq 6 \\ 0 & \text { if } & x<4 \text { and } x>6\end{array}\right.\right.$

Here ${ }^{\alpha} \mu=[\mu(\alpha), \bar{\mu}(\alpha)]=[\alpha+1,3-\alpha]$ and

${ }^{\alpha} \gamma=[\gamma(\alpha), \bar{\gamma}(\alpha)]=[\alpha+4,6-\alpha]$. There is no intersecting points of $\mu(x)$ and $\gamma(x)$ and the $\alpha$-cuts of $M A X\left(\mu_{\gamma} \gamma\right)$ and $M N\left(\mu_{3} \gamma\right)$ is :

$$
{ }^{a}\left[\operatorname{MAX}\left(\mu_{1} \gamma\right)\right]=[\alpha+1,3-\alpha] \text { if }[0,1]
$$


and $\quad[M I N(\mu, \gamma)]=[\alpha+4,6-\alpha]$ if $[0,1]$

Now, from (1) and (2), we obtain the corresponding membership functions of the maximum and the minimum fuzzy numbers of $\mu$ and $\gamma$, which are as follows :

$$
\begin{aligned}
& \operatorname{MAX}(\mu, \gamma)(x)=\left\{\begin{array}{ccc}
x-4 & \text { if } & 4 \leq x \leq 5 \\
6-x & \text { if } & 5 \leq x \leq 6 \\
0 & \text { if } & x \leq 4 \text { and } x>6
\end{array}=\gamma(x)\right. \\
& \text { and } \quad M N(\mu, \gamma)(x)=\left\{\begin{array}{ccc}
x-1 & \text { if } & 1 \leq x \leq 2 \\
3-x & \text { if } & 2 \leq x \leq 3 \\
0 & \text { if } & x<1 \text { and } x>3
\end{array}=\mu(x)\right. \text {. }
\end{aligned}
$$

Example 3.2 : Let us consider the fuzzy numbers $\mu$ and $\gamma$ which are defined by :

$$
\begin{aligned}
& \mu(x)=\left\{\begin{array}{ccc}
x-1 & \text { if } & 1 \leq x \leq 2 \\
3-x & \text { if } & 2 \leq x \leq 3 \\
0 & \text { if } & x \leq 1 \text { and } x 3
\end{array}\right. \\
& \gamma(x)=\left\{\begin{array}{ccc}
x-2 & \text { if } & 2 \leq x \leq 3 \\
4-x & \text { if } & 3 \leq x \leq 4 \\
0 & \text { if } & x<3 \text { and } x>4
\end{array} .\right.
\end{aligned}
$$

and

Here $\quad \alpha_{\mu}=[\underline{\mu}(\alpha), \bar{\mu}(\alpha)]=[\alpha+1,3-\alpha]$ and $a_{\gamma}=[\underline{\gamma}(\alpha), \bar{\gamma}(\alpha)]=[\alpha+2,4-\alpha]$. In this case, we have only one intersecting points of $\mu(x)$ and $\gamma(x)$ which is $\alpha=\frac{1}{2}$. So, the open intervals are $\left(0, \frac{1}{2}\right)$ and $\left(\frac{1}{2}, 1\right)$. Therefore, by hand calculation, the maximum and the minimum of $\mu$ and $\gamma$ are computed, whose membership functions are

$$
M A X(\mu, \gamma)(x)=\gamma(x) \text { and } M I N(\mu, \gamma)(x)=\mu(x) .
$$

Example 3.3 : Consider two fuzzy numbers $\mu$ and $\gamma$ which are defined by

$$
\mu(x)=\left\{\begin{array}{ccc}
\frac{x+2}{3} & \text { if } & -2 \leq x \leq 1 \\
\frac{4-x}{3} & \text { if } & 1 \leq x \leq 4 \\
0 & \text { if } & x<-2 \text { and } x>1
\end{array} ; \quad \gamma(x)=\left\{\begin{array}{ccc}
x-1 & \text { if } & 1 \leq x \leq 2 \\
3-x & \text { if } & 2 \leq x \leq 3 \\
0 & \text { if } & x<1 \text { and } x>3
\end{array} .\right.\right.
$$

So, $\alpha_{\mu}=[\underline{\mu}(\alpha), \bar{\mu}(\alpha)]=[3 \alpha-2,4-3 \alpha]$ and $\alpha_{\gamma}=[\underline{\gamma}(\alpha), \bar{\gamma}(\alpha)]=[\alpha+1,3-\alpha]$. For this case there are only two intersecting points of $\mu(x)$ and $\gamma(x)$ which are at $\alpha_{1}=\frac{1}{2}$ and $\alpha_{2}=\frac{3}{4}$. So, the open intervals are $\left(0, \frac{1}{2}\right),\left(\frac{1}{2}, \frac{3}{4}\right)$ and $\left(\frac{3}{4}, 1\right)$. Therefore, by hand calculation, we obtain 


$$
\begin{array}{r}
\alpha[\operatorname{MAX}(\mu, \gamma)]=\left\{\begin{array}{lll}
{[\alpha+1,4-3 \alpha]} & \text { if } & \alpha \in\left[0, \frac{1}{2}\right] \\
{[\alpha+1,3-\alpha]} & \text { if } & \alpha \in\left[\frac{1}{2}, \frac{3}{4}\right] \\
{[\alpha+1,3-\alpha]} & \text { if } & \alpha \in\left[\frac{3}{4}, 1\right]
\end{array}\right. \\
\text { and }[\operatorname{MIN}(\mu, \gamma)]=\left\{\begin{array}{lll}
{[3 \alpha-2,3-\alpha]} & \text { if } & \alpha \in\left[0, \frac{1}{2}\right] \\
{[3 \alpha-2,4-3 \alpha]} & \text { if } & \alpha \in\left[\frac{1}{2}, \frac{3}{4}\right] \\
{[3 \alpha-2,4-3 \alpha]} & \text { if } & \alpha \in\left[\frac{3}{4}, 1\right]
\end{array}\right.
\end{array}
$$

Now, from (1) and (2), the obtained membership functions of the maximum and the minimum fuzzy numbers of $\mu$ and $\gamma$ are :

$$
\begin{gathered}
M A X(\mu, \gamma)(x)=\left\{\begin{array}{ccc}
x-1 & \text { if } & 1 \leq x \leq 2 \\
3-x & \text { if } & 2 \leq x \leq \frac{5}{2} \\
\frac{4-x}{3} & \text { if } & \frac{5}{2} \leq x \leq 4 \\
0 & \text { if } & x \leq 1 \text { and } x>4
\end{array}\right. \\
M I N(\mu, \gamma)(x)=\left\{\begin{array}{ccc}
\frac{x+2}{3} & \text { if } & -2 \leq x \leq 1 \\
\frac{4-x}{3} & \text { if } & 1 \leq x \leq \frac{5}{2} \\
3-x & \text { if } & \frac{5}{2} \leq x \leq 3 \\
0 & \text { if } & x<-2 \text { and } x>1
\end{array} .\right.
\end{gathered}
$$

Example 3.4 : Let us consider two fuzzy numbers $\mu$ and $\gamma$ which are defined by

$$
\mu(x)=\left\{\begin{array}{ccc}
\frac{x+3}{7} & \text { if } & -3 \leq x \leq 4 \\
\frac{6-x}{2} & \text { if } & 4 \leq x \leq 6 \\
0 & \text { if } & x<-3 \text { and } x>6
\end{array} ; \quad \gamma(x)=\left\{\begin{array}{clc}
x-1 & \text { if } & 1 \leq x \leq 2 \\
\frac{8-x}{6} & \text { if } & 2 \leq x \leq 8 \\
0 & \text { if } & x<1 \text { and } x>8
\end{array} .\right.\right.
$$

We have three intersecting points of $\mu(x)$ and $\gamma(x)$ which are $\alpha_{1}=\frac{1}{2}, \alpha_{2}=\frac{2}{3}$ and $\alpha_{3}=\frac{11}{13}$. So, the open intervals are $\left(0, \frac{1}{2}\right),\left(\frac{1}{2}, \frac{2}{3}\right),\left(\frac{2}{3}, \frac{11}{13}\right)$ and $\left(\frac{11}{13}, 1\right)$. Therefore, by hand calculation, we get the membership functions of the maximum and the minimum fuzzy numbers of $\mu$ and $\gamma$ which are as under 


$$
\begin{gathered}
M A X(\mu, \gamma)(x)=\left\{\begin{array}{clc}
x-1 & \text { if } & 1 \leq x \leq \frac{5}{3} \\
\frac{x+3}{7} & \text { if } & \frac{5}{3} \leq x \leq 4 \\
\frac{6-x}{2} & \text { if } & 4 \leq x \leq 5 \\
\frac{8-x}{6} & \text { if } & 5 \leq x \leq 8 \\
0 & \text { if } & x<1 \text { and } x>8
\end{array}\right. \\
M I N(\mu, \gamma)(x)=\left\{\begin{array}{clc}
\frac{x+3}{7} & \text { if } & -3 \leq x \leq \frac{5}{3} \\
x-1 & \text { if } & \frac{5}{3} \leq x \leq 2 \\
\frac{0-x}{6} & \text { if } & 2 \leq x \leq 5 \\
\frac{6-x}{2} & \text { if } & 5 \leq x \leq 6 \\
0 & \text { if } & x<-3 \text { and } x>6
\end{array}\right.
\end{gathered}
$$

\section{Result obtained from The Computer Program}

It is true that one might have obtained the membership functions of the maximum and the minimum of two triangular fuzzy numbers by hand calculation, but the process is lengthy and time consuming. Here we have created a programming code in order to show the membership functions of maximum and minimum fuzzy numbers.

The program has the ability to fulfill the requirement as proposed. It is able to read the fuzzy numbers $\mu(x)$ and $\gamma(x)$; and can show the analytic expressions of the optimization functions and the corresponding graphical representations. Researchers are using fuzzy numbers in solving problems based on applications in artificial intelligence, decision making and so on, by comparing fuzzy numbers, because of their suitability for representing uncertain information, such as, to control traffic signal, to diagnose of diseases etc. In these contexts, comparing fuzzy numbers is essential and it is also used in optimization problem. Therefore, a quicker tool is necessary to obtain a total ordering or partial ordering of fuzzy numbers. Now, we need to observe how the program works.

Let us consider the fuzzy numbers $\mu$ and $\gamma$ which are defined in the examples of Section 3. After executing the program, the obtained outputs are given below.

The analytical expressions of the maximum and the minimum fuzzy numbers, $\operatorname{MAX}(\mu, \gamma)$ and $\operatorname{MIN}(\mu, \gamma)$ respectively, are shown in the following table : 
Table : Analytical expressions $M A X(\mu, \gamma)$ and $M I N(\mu, \gamma)$ of two fuzzy numbers, respectively

\begin{tabular}{|c|c|c|}
\hline & $\operatorname{MAX}(\mu, y)(x)$ & $M N(\mu, y)(x)$ \\
\hline 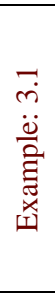 & $\begin{array}{l}\text { 0, otherwise } \\
\left\{\left\{-4+x,\left\{\left\{x \rightarrow \text { Interval }\left[\left\{\frac{7}{2}, 4\right\}\right]\right\}\right\}\right\},\right. \\
\left\{6-x,\left\{\left\{x \rightarrow \text { Interval }\left[\left\{6, \frac{13}{2}\right\}\right]\right\}\right\}\right\}, \\
\{-4+x,\{\{x \rightarrow \text { Interval }[\{4,5\}]\}\}\}, \\
\{6-x,\{\{x \rightarrow \text { Interval }[\{5,6\}]\}\}\}\}\end{array}$ & $\begin{array}{l}\text { 0, otherwise } \\
\left\{\left\{-1+x,\left\{\left\{x \rightarrow \text { Interval }\left[\left\{\frac{1}{2}, 1\right\}\right]\right\}\right\}\right\},\right. \\
\left\{3-x,\left\{\left\{x \rightarrow \text { Interval }\left[\left\{3, \frac{7}{2}\right\}\right]\right\}\right\}\right\}, \\
\{-1+x,\{\{x \rightarrow \text { Interval }[\{1,2\}]\}\}\}, \\
\{3-x,\{\{x \rightarrow \text { Interval }[\{2,3\}]\}\}\}\}\end{array}$ \\
\hline 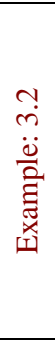 & $\begin{array}{l}\text { 0, otherwise } \\
\left\{\left\{-2+x,\left\{\left\{x \rightarrow \text { Interval }\left[\left\{2, \frac{5}{2}\right\}\right]\right\}\right\}\right\},\right. \\
\left\{4-x,\left\{\left\{x \rightarrow \text { Interval }\left[\left\{\frac{7}{2}, 4\right\}\right]\right\}\right\}\right\}, \\
\left\{-2+x,\left\{\left\{x \rightarrow \text { Interval }\left[\left\{\frac{5}{2}, 3\right\}\right]\right\}\right\}\right\}, \\
\left.\left\{4-x,\left\{\left\{x \rightarrow \text { Interval }\left[\left\{3, \frac{7}{2}\right\}\right]\right\}\right\}\right\}\right\}\end{array}$ & $\begin{array}{l}\text { 0, otherwise } \\
\left\{\left\{-1+x,\left\{\left\{x \rightarrow \text { Interval }\left[\left\{1, \frac{3}{2}\right\}\right]\right\}\right\}\right\},\right. \\
\left\{3-x,\left\{\left\{x \rightarrow \text { Interval }\left[\left\{\frac{5}{2}, 3\right\}\right]\right\}\right\}\right\}, \\
\left\{-1+x,\left\{\left\{x \rightarrow \text { Interval }\left[\left\{\frac{3}{2}, 2\right\}\right]\right\}\right\}\right\}, \\
\left.\left\{3-x,\left\{\left\{x \rightarrow \text { Interval }\left[\left\{2, \frac{5}{2}\right\}\right]\right\}\right\}\right\}\right\}\end{array}$ \\
\hline 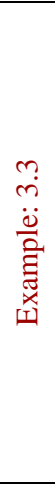 & $\begin{array}{l}\text { 0, otherwise } \\
\left\{\left\{-1+x,\left\{\left\{x \rightarrow \text { Interval }\left[\left\{1, \frac{3}{2}\right\}\right]\right\}\right\}\right\},\right. \\
\left\{\frac{4-x}{3},\left\{\left\{x \rightarrow \text { Interval }\left[\left\{\frac{5}{2}, 4\right\}\right]\right\}\right\}\right\}, \\
\left\{-1+x,\left\{\left\{x \rightarrow \text { Interval }\left[\left\{\frac{3}{2}, \frac{7}{4}\right\}\right]\right\}\right\}\right\}, \\
\left\{3-x,\left\{\left\{x \rightarrow \text { Interval }\left[\left\{\frac{9}{4}, \frac{5}{2}\right\}\right]\right\}\right\}\right\}, \\
\left\{-1+x,\left\{\left\{x \rightarrow \text { Interval }\left[\left\{\frac{7}{4}, 2\right\}\right]\right\}\right\}\right\}, \\
\left.\left\{3-x,\left\{\left\{x \rightarrow \text { Interval }\left[\left\{2, \frac{9}{4}\right\}\right]\right\}\right\}\right\}\right\}\end{array}$ & $\begin{array}{l}\text { 0, otherwise } \\
\left\{\left\{\frac{2+x}{3},\left\{\left\{x \rightarrow \text { Interval }\left[\left\{-2,-\frac{1}{2}\right\}\right]\right\}\right\}\right\},\right. \\
\left\{3-x,\left\{\left\{x \rightarrow \text { Interval }\left[\left\{\frac{5}{2}, 3\right\}\right]\right\}\right\}\right\}, \\
\left\{\frac{2+x}{3},\left\{\left\{x \rightarrow \text { Interval }\left[\left\{-\frac{1}{2}, \frac{1}{4}\right\}\right]\right\}\right\}\right\}, \\
\left\{\frac{4-x}{3},\left\{\left\{x \rightarrow \text { Interval }\left[\left\{\frac{7}{4}, \frac{5}{2}\right\}\right]\right\}\right\}\right\}, \\
\left\{\frac{2+x}{3},\left\{\left\{x \rightarrow \text { Interval }\left[\left\{\frac{1}{4}, 1\right\}\right]\right\}\right\}\right\}, \\
\left.\left\{\frac{4-x}{3},\left\{\left\{x \rightarrow \text { Interval }\left[\left\{1, \frac{7}{4}\right\}\right]\right\}\right\}\right\}\right\}\end{array}$ \\
\hline 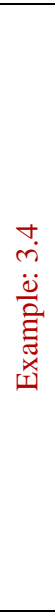 & $\begin{array}{l}\text { 0, otherwise } \\
\left\{\left\{-1+x,\left\{\left\{x \rightarrow \text { Interval }\left[\left\{1, \frac{3}{2}\right\}\right]\right\}\right\}\right\},\right. \\
\left\{\frac{8-x}{6},\{\{x \rightarrow \text { Interval }[\{5,8\}]\}\}\right\}, \\
\left\{-1+x,\left\{\left\{x \rightarrow \text { Interval }\left[\left\{\frac{3}{2}, \frac{5}{3}\right\}\right]\right\}\right\}\right\}, \\
\left\{\frac{6-x}{2},\left\{\left\{x \rightarrow \text { Interval }\left[\left\{\frac{14}{3}, 5\right\}\right]\right\}\right\}\right\}, \\
\left\{\frac{3+x}{7},\left\{\left\{x \rightarrow \text { Interval }\left[\left\{\frac{5}{3}, \frac{38}{13}\right\}\right]\right\}\right\}\right\}, \\
\left\{\frac{6-x}{2},\left\{\left\{x \rightarrow \text { Interval }\left[\left\{\frac{56}{13}, \frac{14}{3}\right\}\right]\right\}\right\}\right\}, \\
\left\{\frac{3+x}{7},\left\{\left\{x \rightarrow \text { Interval }\left[\left\{\frac{38}{13}, 4\right\}\right]\right\}\right\}\right\}, \\
\left.\left\{\frac{6-x}{2},\left\{\left\{x \rightarrow \text { Interval }\left[\left\{4, \frac{56}{13}\right\}\right]\right\}\right\}\right\}\right\}\end{array}$ & $\begin{array}{l}\text { 0, otherwise } \\
\left\{\left\{\frac{3+x}{7},\left\{\left\{x \rightarrow \text { Interval }\left[\left\{-3, \frac{1}{2}\right\}\right]\right\}\right\}\right\},\right. \\
\left\{\frac{6-x}{2},\{\{x \rightarrow \text { Interval }[\{5,6\}]\}\},\right. \\
\left\{\frac{3+x}{7},\left\{\left\{x \rightarrow \text { Interval }\left[\left\{\frac{1}{2}, \frac{5}{3}\right\}\right]\right\}\right\}\right\}, \\
\left\{\frac{8-x}{6},\{\{x \rightarrow \text { Interval }[\{4,5\}]\}\},\right. \\
\left\{-1+x,\left\{\left\{x \rightarrow \text { Interval }\left[\left\{\frac{5}{3}, \frac{24}{13}\right\}\right]\right\}\right\}\right\}, \\
\left\{\frac{8-x}{6},\left\{\left\{x \rightarrow \text { Interval }\left[\left\{\frac{38}{13}, 4\right\}\right]\right\}\right\}\right\}, \\
\left\{-1+x,\left\{\left\{x \rightarrow \text { Interval }\left[\left\{\frac{24}{13}, 2\right\}\right]\right\}\right\}\right\}, \\
\left.\left\{\frac{8-x}{6},\left\{\left\{x \rightarrow \text { Interval }\left[\left\{2, \frac{38}{13}\right\}\right]\right\}\right\}\right\}\right\}\end{array}$ \\
\hline
\end{tabular}

The program has generated the graphical representations of Fuzzy numbers, discussed in Examples 3.1, 3.2, 3.3, and 3.4, are shown in Figure 1. 


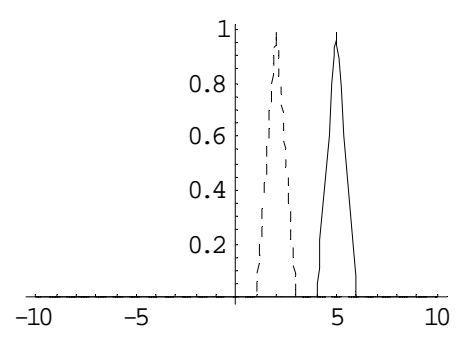

(a) Graphs from Example 3.1

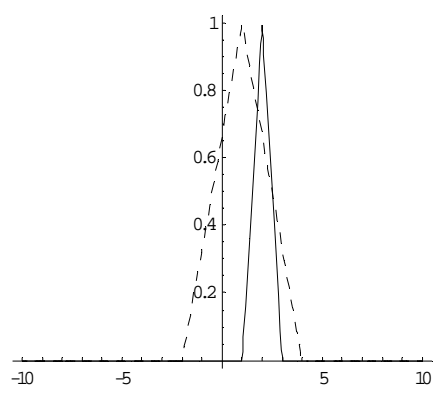

(c) Graphs from Example 3.3

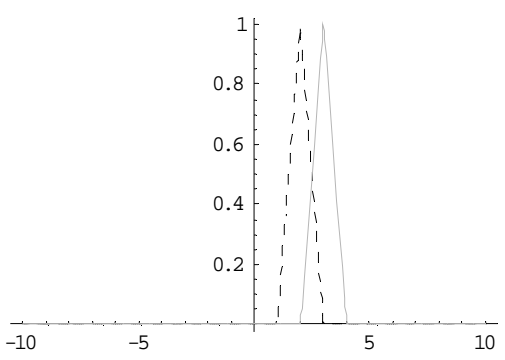

(b) Graphs from Example 3.2

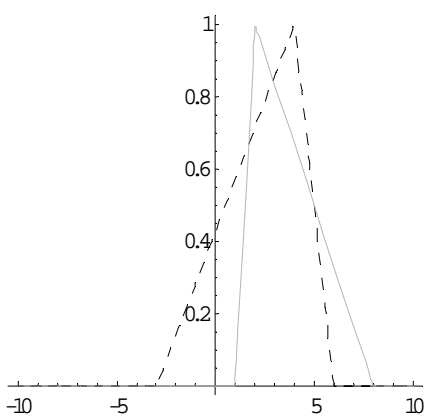

(d) Graphs from Example 3.4

Figure 1: Graphical representations of Fuzzy numbers, shown in Examples 3.1, 3.2, 3.3, and 3.4.

By using the program, the outputs of the corresponding graphical representations of fuzzy numbers $\operatorname{MAX}(\mu, \gamma)(x)$, from Figure.1 (a), (b), (c), (d), respectively, are in Figure 2.
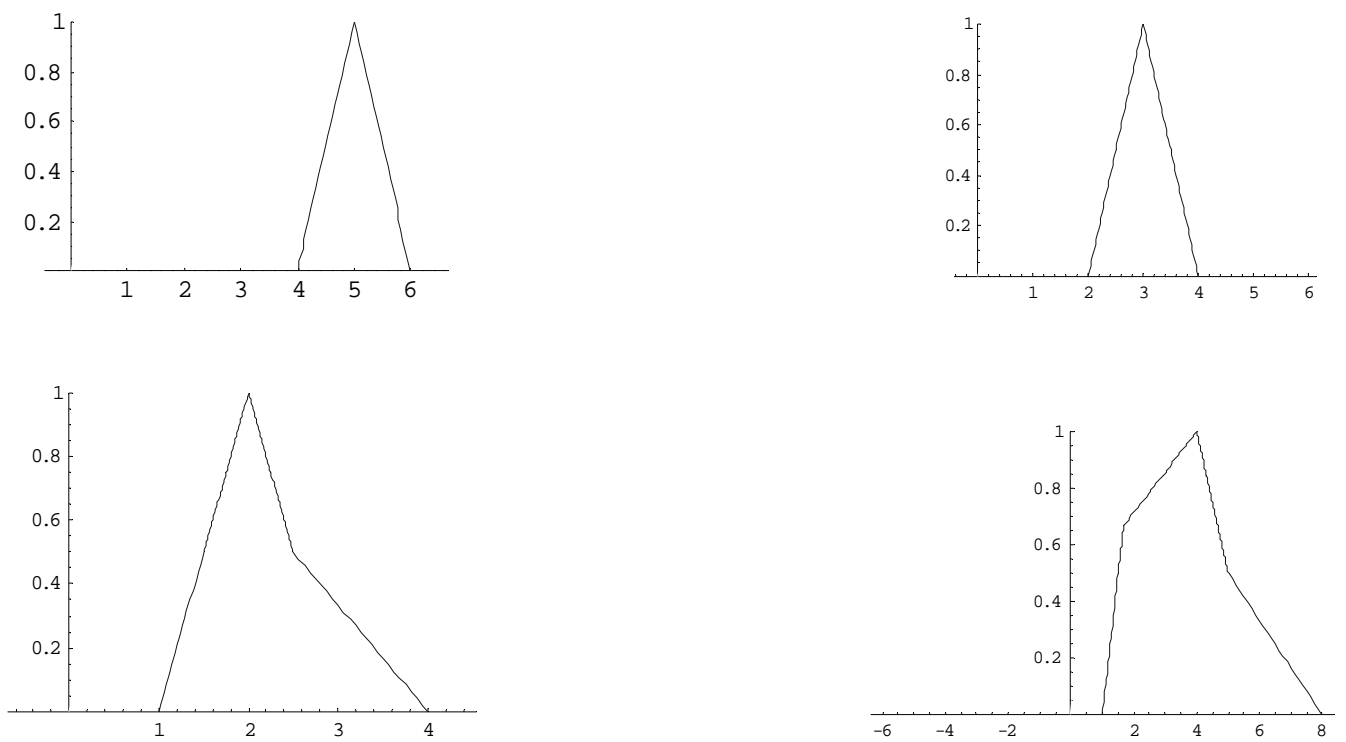

Figure 2 : Graphical representations of Fuzzy numbers $\operatorname{MAX}(\boldsymbol{\alpha}, \gamma)(x)$.

Figure. 3 describes the outputs of the graphical representations of Fuzzy numbers $\operatorname{MIN}(\mu, \gamma)(x)$, from Figure.1 (a), (b), (c), (d) respectively, by using the program. 


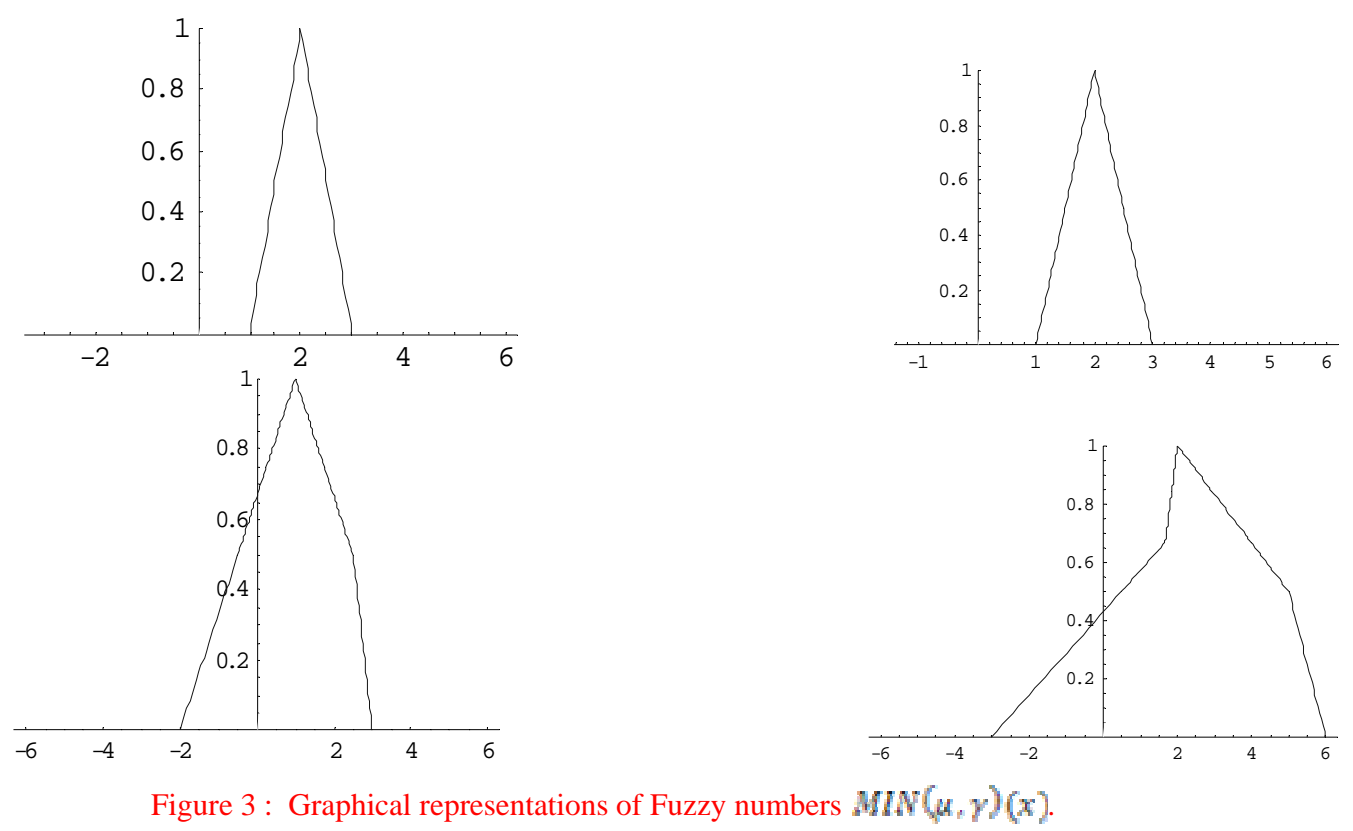

\section{Generating MAX and MIN of More Than Two Fuzzy Numbers}

Choose the fuzzy number $\eta_{1}=\operatorname{MAX}(\mu, \gamma)$ and a new fuzzy number $\eta_{2}$. Then, by using the program we are able to get the maximum of three fuzzy numbers $\mu, \gamma$, and $\eta_{2}$. In a similar way, it is possible to obtain the minimum of three fuzzy numbers $\mu, \gamma$, and $\eta_{2}$. Therefore, by continuing this process, the maximum and the minimum of more than two fuzzy numbers, what ever we like, can be found.

\section{Conclusion}

In this paper we have presented an efficient and interesting algorithm to represent the membership functions of the maximum fuzzy number and the minimum fuzzy number of any two or more than two triangular fuzzy numbers to invent an optimum fuzzy system by comparing fuzzy numbers. It may be mentioned here, fuzzy set theory is more essential to the researchers who love to use it in practical problems, such as decision making problem by comparing fuzzy data. In order to that, a computer program is created by using CAS (MATHEMATICA) to obtain analytic expressions and the corresponding graphical representations of $\operatorname{MAX}\left(\mu_{s} \gamma\right)(x)$ and $\operatorname{MIN}\left(\mu_{n} \gamma\right)(x)$. Though many works have already been done on the fuzzy operators $M A X$ and $M I N$, but none took attempt to work on the proposed algorithm and program so that a model can be developed by using fuzzy ordered relation on the set of fuzzy numbers. The proposed programming code can be extended to read the trapezoidal and the other fuzzy numbers to obtain the desired results. It is obvious that no new innovation or research work is capable of solving all the requirements. In this paper an algorithm is established to give a basic notion. Therefore, more work can be done 


\section{following this idea and the limitation can easily be overcome. \\ REFERENCES}

1. Boukezzoula, R., Galichet,S., Foulloy, L. MIN and MAX Operators for Fuzzy Intervals and Their Potential use in Aggregation Operators, IEEE Transactions on Fuzzy Systems, Vol. 15, No. 6, December 2007, p. 1135-1144.

2. Chiu, C. H., Wang, W. J., A Simple Computation of MIN and MAX Operations for Fuzzy Numbers, Fuzzy Sets and Systems, 126, (2002), p. 273-276.

3. Dubois, D., Prade, H., Operations on Fuzzy Numbers, Internet. J. Systems Science, 9(6), (1978), p. 613626.

4. HONG, D. H, and KIM, K. T., An Easy Computation of MIN and MAX Operations for Fuzzy Numbers, J. Appl. Math. \& Computing, Vol. 21, (2006), No. 1-2, p. 555-561.

5. Kaufmann, A., Gupta M. M., Introduction to Fuzzy Arithmetic Theory and Applications, Van Nostrand Reinhold Company Inc., (1985), p. 1-43.

6. Klir, G. J., Yua, B., Fuzzy Sets and Fuzzy Logic Theory and Applications, Prentice-Hall of India Private Limited, New Delhi, (1997), p. 1-117.

7. Saade, J. J, and Schwarzlander, H., Ordering Fuzzy Sets over real line : an approach based on decision making under uncertainty, Fuzzy Sets and Systems, Vol.50, (1992), p. 237-246.

8. Shirin, S., A Method to Find The Membership Functions of Maximum and Minimum of Fuzzy Numbers, Ganit, J. Bangladesh Math. Soc., Vol.30 (2010), p. 89-99.

9. Tanaka, K., An Introduction to Fuzzy Logic for Practical Applications, Springer-Verlag, New York, 1997. P. 5-50.

10. Zadeh, L. A., Fuzzy Sets, Information and Control, 8(3), (1965), p. 338-353. 